\title{
Vertebral Artery Occlusive Disease: Data from the angiographically confirmed vertebral artery (ACVAD) disease registry
}

\author{
Sehar Babar MD'1,Omar Saeed MD ${ }^{1}$, Adnan I. Qureshi MD ${ }^{1}$ \\ ${ }^{1}$ Zeenat Qureshi Stroke Institute, St. Cloud, MN, USA
}

\section{BACKGROUND}

Vertebral artery occlusive disease (VAOD), although equally prevalent as internal carotid artery occlusive disease, remains understudied. We performed this study to identify basic demographic and clinical characteristics of adult patients with angiographically confirmed VAOD.

\section{METHODS}

All patients aged 18 years or older who had angiographically confirmed (using catheter based angiography to ensure diagnostic accuracy) VAOD, defined as stenosis of $50 \%$ or greater, were included. The demographic and clinical characteristics (including associated clinical symptoms), and angiographic features including severity and location of stenosis, and collateral pathways were ascertained using predefined criteria. The protocol for data collection was approved by local Institutional Review Board and informed consent was waivered. All cerebral angiographic procedures were performed by one operator (AIQ). Data from angiographic images; 1) the severity of stenosis; 2) side of stenosis (right or left); 3) location (intracranial which included dural location and extracranial disease); 4) characteristics of lesion (regular or irregular); and 5) presence of collaterals (retrograde filling from contralateral side, or anterograde filling from anastomoses by ipsilateral ascending cervical artery or occipital artery). The severity of intracranial stenosis was measured using the Warfarin-Aspirin Symptomatic Intracranial Disease (WASID) criteria and extracranial stenosis was measured using a previously described method. ${ }^{1}$
Table 1. Baseline demographic and clinical characteristics and stroke related data of patients with and without vertebrobasilar insufficiency.

\begin{tabular}{|c|c|c|c|}
\hline & Symptomatic & $\begin{array}{l}\text { Asymptom } \\
\text { atic }\end{array}$ & $\begin{array}{l}p- \\
\text { value }\end{array}$ \\
\hline Overall number (\%) & 22 & 34 & \\
\hline Age & $66.7 \pm 11.7$ & $65.4 \pm 13.2$ & 0.7 \\
\hline \multicolumn{4}{|l|}{ Gender } \\
\hline Men & $12(54.5 \%)$ & $19(55.9 \%)$ & \\
\hline Women & $10(45.5 \%)$ & $15(44.1 \%)$ & 0.9 \\
\hline \multicolumn{4}{|l|}{ Race/Ethnicity } \\
\hline Hispanic & $3(13.6 \%)$ & $1(2.9 \%)$ & \multirow{3}{*}{0.4} \\
\hline White & $17(77.3 \%)$ & $30(88.2 \%)$ & \\
\hline African American & $2(9.1 \%)$ & $2(5.9 \%)$ & \\
\hline \multicolumn{4}{|l|}{ Headaches } \\
\hline Migraine & $3(15 \%)$ & $2(11.8 \%)$ & \\
\hline Non migraine & $2(10 \%)$ & $15(88.2 \%)$ & .024 \\
\hline Acute infarct on neuroimaging & $9(40.9 \%)$ & $0(0 \%)$ & $<.0001$ \\
\hline Recurrent ischemic symptoms & $14(70.0 \%)$ & 0 & $<.0001$ \\
\hline Transient ischemic attack & $8(36.4 \%)$ & 0 & $<.0001$ \\
\hline Ischemic stroke & $14(63.6 \%)$ & 0 & $<.0001$ \\
\hline \multicolumn{4}{|l|}{ Laterality of disease } \\
\hline Unilateral & $13(59.1 \%)$ & $31(91.2 \%)$ & \multirow{2}{*}{.004} \\
\hline Bilateral & $9(40.9 \%)$ & $3(8.8 \%)$ & \\
\hline \multicolumn{4}{|l|}{ Right vertebral artery involvement } \\
\hline Intracranial & $1(4.5 \%)$ & $2(5.6 \%)$ & \multirow{4}{*}{0.7} \\
\hline Extracranial & $10(47.1 \%)$ & $15(44.1 \%)$ & \\
\hline Both & $1(4.5 \%)$ & $0(0 \%)$ & \\
\hline Complete occlusion & $1(4.5 \%)$ & $2(5.9 \%)$ & \\
\hline \multicolumn{4}{|l|}{ Left vertebral artery involvement } \\
\hline Intracranial & $2(9.1 \%)$ & 0 & \multirow{4}{*}{.01} \\
\hline Extracranial & $8(36.4 \%)$ & $17(50.0 \%)$ & \\
\hline Both & $1(4.5 \%)$ & 0 & \\
\hline Complete occlusion & $6(27.3 \%)$ & $4(19.0 \%)$ & \\
\hline \multicolumn{4}{|l|}{ Stenosis severity } \\
\hline Moderate & $4(18.2 \%)$ & $17(50.0 \%)$ & \\
\hline Severe & $5(22.7 \%)$ & $4(11.8 \%)$ & \\
\hline Occluded & $13(59.1 \%)$ & $13(38.2 \%)$ & .04 \\
\hline Percent stenosis ( \pm SD) & $88.1 \pm 16.5$ & $75.4 \pm 20.8$ & .02 \\
\hline Collaterals & & $\perp$ & 1 \\
\hline $\begin{array}{l}\text { Retrograde filling from ascending } \\
\text { cervical and occipital artery }\end{array}$ & 0 & $5(14.7 \%)^{\dagger}$ & \multirow{2}{*}{.02} \\
\hline $\begin{array}{l}\text { Filling from contralateral vertebral } \\
\text { artery }\end{array}$ & $11(50.0 \%)$ & $7(20.6 \%)$ & \\
\hline
\end{tabular}

$\uparrow-3$ from ascending cervical; 1 from occipital artery; 1 from both ascending cervical and occipital arteries

\section{RESULTS}

A total of 56 patients with VAOD confirmed by cerebral angiography were identified. The mean patient age was $65.4 \pm$ SD 11.7 years; $55.4 \%$ were men.

\section{Clinical and imaging characteristics}

VAOD was considered symptomatic in 22 $(39.3 \%)$ of 56 patients; 8 and 14 had transient ischemic attack or ischemic stroke, respectively (Table 1). Recurrent ischemic symptoms were seen in 14 of 22 patients, Of the 56 patients, 9 patients had acute infarction on neuroimaging in vertebral or basilar artery distribution within preceding 3 months. Overall, 45 of the 56 patients with VAOD were using one or more antiplatelet agents. Twenty patients had concurrent diagnosed coronary artery disease. Eight patients underwent angioplasty and/or stent placement.

\section{Angiographic features}

Of 56 patients with VAOD, $37.5 \%$ were classified as suffering from moderate stenosis $(50-69 \%)$, $16.1 \%$ from severe stenosis $(70-99 \%)$ and $46.4 \%$ from occlusion of at least one vertebral artery. The VAOD was unilateral and bilateral in 44 and 12 patients, respectively. Collateral flow was seen in 23 patients either through anastomoses of distal vertebral artery with ascending cervical artery or occipital artery $(n=5)$ or retrograde filling from contralateral vertebral artery $(n=18)$. Nineteen had stenosis $\geq 50 \%$ or occlusion in the internal carotid artery.

There was a significantly higher frequency of bilateral vertebral artery disease in patients with ischemic symptoms $(40.9 \%$ versus $8.8 \%$, $\mathrm{p}=0.004$, see Table 1$)$. The severity of stenosis (\% with SD) was significantly greater in patients with ischemic symptoms (88.1 \pm 16.5 versus $75.4 \pm 20.8, p=0.02)$. The proportion of patients with severe stenosis or occlusion was higher in patients with ischemic symptoms $(82 \%$ versus $50 \%, \quad p=.04$.) Anterograde filling from anastomoses by ipsilateral ascending cervical artery or occipital artery was more common in patients with ischemic symptoms.

\section{CONCLUSION}

Registries such as ACVAD are of unique importance in order to provide better understanding of asymptomatic and symptomatic VAOD and assist in designing future clinical trials.

Reference:

1. Samuels OB, Joseph GJ, Lynn MJ, et al. A standardized method for measuring intracranial arterial stenosis. AJNR American journal of neuroradiology. 2000;21(4):643-6. 